Editorial:

\title{
The Prehospital EMS Provider in the 1990 s: The Third Decade of Paramedics
}

Field paramedics have been around for almost 20 years. The growth and development of paramedic programs during the last two decades have been analogous to childhood and adolescence. In the third decade of the paramedic, additional major changes will be more subtle: akin to the maturation of early adulthood.

Currently, we are witnessing a transition from the rote training of paramedics to serve as technicians towards a more sophisticated educational process resulting in the development of the field technologist. This transition is the direct result of the growing trend toward academics which gradually is pervading the EMS systems in the United States and in many other countries. In part, this is evidenced through the strong support by the EMS community for Prehospital and Disaster Medicine and by the success of its precursors from the Acute Care Foundation (Jourmal of Prehospital Care, Tampa BayEMS Journal) and the World Association for Emergency and Disaster Medicine (Journal of Emergency and Disaster Medicine).

As more sophisticated monitoring and treatment technologies become available for use in the prehospital environment, an enhanced capability to initiate definitive therapy prior to arrival at the hospital will become both possible and practical. Advances in medicine typically follow improvements in monitoring technology. However, introduction of a specific treatment modality or technology into the prehospital environment is worthy of consideration only when: 1) it is likely to impact significantly and positively on both mortality and morbidity; 2) it is best implemented early in the acute course of care; and 3) its implementation is both cost-effective and has demonstrated positive impact on cost-benefit. The most significant factors which will limit augmentation of the scope of prehospital emergency care will be the quality and intensity of the paramedic curricula, the continuing education available, and character of the medical control provided. Without optimization of these three factors, the more sophisticated techniques for monitoring and treatment will never gain acceptance in prehospital emergency medical services.

Fueled by the potential for earlier definitive care and the potential for reduced morbidity and mortality, paramedic education programs are likely to follow a course similar to that which has occurred in nursing. Certificate programs slowly may give way to associate and baccalaureate degree programs. A baccalaureate degree will become the standard for promotion into a management or educator position. Post-graduate education will become the norm for upper-level management and collegiate EMS faculty positions.

In order to realize a more sophisticated level of EMS, newer mechanisms for the provision of appropriate basic and post-graduate and continuing education resources must be developed. Along with the evolution of video and computer communications technology, the logistics of the educational process will become much more convenient. These media will provide access to continuing education resources and electronic college classrooms within our substations and homes. This will alleviate many of the problems associated with return to the academic setting while still working full time, supporting a family, etc. Self-directed continuing education and formal course work will become commonplace with interactive video, on-line and electronic mail access to instructors, and on-line medical seminars on a an international scale. This technology will be advantageous particularly to volunteer and rural EMS personnel 
who tend to have greater obstacles which until now have made it difficult to take advantage of quality educational resources.

An important co-requisite to these changes in our educational and clinical practices will be the development and implementation of research. Valid research will be needed to substantiate the purported benefits of proposed newer educational and clinical methods. The more advanced educational background of the paramedics of the future will enable them to become legitimate collaborators with EMS physicians in the conduct of such research.

In addition, the issues associated with career ladders for paramedics must be addressed. With increased public expectations of field personnel, a means to encourage and compensate personnel who seek and achieve these higher goals must be provided. Positions may be created for the longer terms of instruction and supervision of paramedic internships, for designated research crew appointments, for CME faculty appointments, and for pay categorization based on certificate, associate, baccalaureate, and post-graduate education. Development of professional EMS career ladders for EMS personnel within the fire service will pose special administrative challenges. In all to many cases, the only way to promotion in a fire department is to become a traditional fire officer in which clinical responsibilities become relegated to supervision of the scene. The situation isn't much better in most third service EMS agencies. Administrative research will be needed to solve these problems and the postgraduate training of the future EMS administrators must provide the background necessary.

Between now and then, someone must build these programs and pioneer these technologies-and that future is in your hands.

Michael R. Gunderson, REMT-P

President, Acute Care Foundation

Pinellas County EMS System, Palm Harbor Fire Department

Tampa, Florida, USA 